\title{
LEARN - Um novo protocolo de roteamento para VANET baseado em setorização hexagonal
}

\author{
Celso A. R. L. Brennand ${ }^{1}$, Geraldo P. Rocha Filho ${ }^{2}$, Rodolfo I. Meneguette ${ }^{3}$ \\ ${ }^{1}$ Universidade Federal Rural de Pernambuco (UFRPE), \\ Unidade Acadêmica de Serra Talhada (UAST) \\ ${ }^{2}$ Departamento de Ciência da Computação, Universidade de Brasília (UnB), \\ Campus Darcy Ribeiro, Brasília, DF, Brasil \\ ${ }^{3}$ Instituto De Ciências Matemáticas e de Computação, Universidade de São Paulo (USP) \\ celso.brernnand@ufrpe.br, geraldof@unb.br, meneguette@icmc.usp.br
}

\begin{abstract}
One of the main problems with the route suggestion services provided by the Intelligent Transport System (ITS) concerns communication, such as network delay, disconnection, and coverage. With that in mind, this article proposes LEARN, a routing protocol for Vanet based on hexagonal sectorization. To this end, LEARN sectors the scenario into hexagons associating vehicles to sectors according to their location. Thus, messages are forwarded between sectors to their destination, taking advantage of the knowledge of the densities in each region. Therefore, LEARN adapts to the inherent characteristics of the environment, such as the density of regions and vehicle flow. When compared to other related works, LEARN presents a reduction in the number of messages of 95\%, maintaining coverage at $97 \%$ in different scenario characteristics.
\end{abstract}

Resumo. Um dos principais problemas dos serviços de sugestão de rotas providos pelo Sistema de Transporte Inteligente (ITS) diz respeito a comunicação, tais como o atraso da rede, desconexão e cobertura. Com isso em mente, este artigo propõe o LEARN, um protocoLo de rotEamento para vAnet baseado em setoRização hexagoNal. Para tanto, o LEARN setoriza o cenário em hexágonos associando os veículos aos setores de acordo com sua localização. Com isso, as mensagens são encaminhadas entre os setores até o destino tirando proveito do conhecimento das densidades em cada região. Portanto, o LEARN se adapta às características inerentes do ambiente, como a densidade de regiões e fluxo de veículos. Quando comparado com outros trabalhos relacionados, o LEARN apresenta uma redução no número de mensagens de 95\%, mantendo a cobertura em $97 \%$ em diferentes características de cenário.

\section{Introdução}

Os veículos podem ser equipados com dispositivos embarcados que atendem a inúmeros serviços e aplicações de gestão de tráfego rodoviário ou de grandes cidades [Rodrigues et al. 2019]. Tais dispositivos permitem o monitoramento das ruas e rodovias de uma cidade, trazendo novos serviços e auxiliando o Sistema de Transporte Inteligente (ITS), no qual visa otimizar a infraestrutura do sistema de transporte de uma cidade [Meneguette et al. 2018]. Diante do potencial do uso dos aplicativos e serviços 
providos pelo ITS, tomamos como exemplo o estudo realizado pela Organização Mundial da Saúde [Organization et al. 2018]. O estudo apresenta que o número de acidentes fatais em rodovias ao redor do mundo é de 1,35 milhão de mortos por ano. O custo estimado causado por acidentes fatais e não fatais para a economia mundial é de aproximadamente US\$ 1,8 trilhão [Chen et al. 2019]. Portanto, tais acidentes podem ser reduzidos por meio de dispositivos e mecanismos que monitoram o tráfego e detectam possíveis eventos nas estradas.

Para realizar o monitoramento das condições de tráfego de uma via pública, utiliza-se de um mecanismo no qual se verifica a densidade dos veículos nas vias, sua direção e velocidade [De Souza et al. 2017]. Este mecanismo visa detectar quais vias no trânsito estão lentas, com possíveis pontos de congestionamento ou interditadas, permitindo que os motoristas evitem os congestionamentos [Correa et al. 2014]. É valido salientar que a falta de um sistema de monitoramento de tráfego eficaz impacta significativamente nos orçamentos das nações. O custo estimado do congestionamento de tráfego na União Europeia é de aproximadamente $2 \%$ do seu produto interno bruto (PIB) [H. Allen and Stonehill 2013]. Já nos Estados Unidos são gasto mais de 160 bilhões de dólares por ano [Texas Transportation Institute 2015].

Para resolver o problema de congestionamento, é necessário investir em pesquisas computacionais que forneçam a infraestrutura necessária para suprir o sistema de transporte. Para isso, é imprescindível o desenvolvimento de tecnologias de informação e comunicação eficientes, capazes de proporcionar a integração de veículos [Silva et al. 2019] por meio da comunicação entre Veículos (V2V) e entre Veículos e a Infraestrutura (V2I) rodoviária. As Redes Veiculares Ad-hoc (VANETs) têm como um dos principais problemas as partições e desconexões da rede devido às características das VANETs que possuem uma topologia altamente dinâmica. Entre estes problemas, os serviços de sugestão de rota possuem como desafios reduzir a redundância das informações na rede e o atraso das mensagens [Srivastava et al. 2020b].

Diversas abordagens da literatura detectam e reduzem áreas congestionadas em ambientes urbanos por meio das redes veiculares [Lourenço et al. 2019, Brennand et al. 2017, da Costa et al. 2019]. Em [da Costa et al. 2019], é proposto um protocolo de comunicação em VANETs para sugestões de rota. Entretanto, o protocolo proposto sofre a necessidade de um alto grau de conhecimento da rede. A solução [Brennand et al. 2017] usa a VANET para a comunicação entre os veículos e com a infraestrutura localizada na lateral da estrada (RSU-Road-Side Unit) para calcular sua velocidade, localização e destino dos veículos. Embora essa solução pareça confiável, ela enfrenta muitos desafios de comunicação, tais como dados redundantes e atraso na entrega das informações.

Nesse sentido, este trabalho propõe um novo protocolo VANET denominado LEARN - protocoLo de rotEamento para vAnet baseado em setoRização hexagoNal. O principal diferencial do LEARN é obter maior cobertura ao serviço de sugestão de rota de forma eficiente e confiável mesmo quando os veículos não estejam dentro do raio de cobertura da infraestrutura. Para tanto, o LEARN setoriza o cenário em hexágonos associando os veículos aos setores de acordo com sua localização. Com isso, as mensagens são encaminhadas entre os setores até o destino tirando proveito do conhecimento das densidades em cada região. Esta estratégia permite que LEARN funcionem de forma 
mais eficiente, aumentando a taxa de entrega e cobertura da rede, ao mesmo tempo que reduz a latência de entrega das mensagens. Os resultados das simulações comprovam a eficiência do LEARN quando comparado com outras soluções consagradas da literatura por apresentar quatro resultados promissores, sendo eles: (i) redução do número de mensagens retransmitidas em 97\%; (ii) redução no número de colisões em 99\%; (iii) aumento na cobertura de $98 \%$; e (iv) aumento de $79 \%$ nas mensagens recebidas.

O restante deste trabalho está organizado da seguinte forma. A Seção 2 apresenta os trabalhos relacionados. A Seção 3 descreve como foi modelado o LEARN. A Seção 4 apresenta a metodologia utilizada para validar o LEARN e os resultados obtidos. Por fim, a Seção 5 apresenta a conclusão e os trabalhos futuros.

\section{Trabalhos Relacionados}

As aplicações de ITSs usam as VANETs para enviar mensagens de uma fonte (i.e., veículo ou RSU) para todos os veículos localizados dentro de uma região geográfica [da Cunha et al. 2016]. Essa atividade é conhecida como disseminação de dados e há dois desafios que são explorados: O flooding ocorre quando muitos veículos próximos tentam se comunicar ao mesmo tempo, aumentando drasticamente a colisão de mensagens [Tonguz et al. 2010, Schwartz et al. 2011]. O problema da conexão intermitente ocorre quando o número de veículos é reduzido (por exemplo, durante o amanhecer, feriados e em áreas rurais), afetando as mensagens de dados disseminadas usando as VANETs [Viriyasitavat et al. 2011, Ros et al. 2012]. Além desses desafios, cada aplicação possui requisitos específicos que demandam diferentes estratégias para apoiar a disseminação dos dados. Muitas soluções de disseminação de dados foram propostas para enfrentar esses desafios [da Cunha et al. 2016, Villas et al. 2014, da Costa et al. 2019, Bao et al. 2020, Srivastava et al. 2020a].

Bao et al. [Bao et al. 2020] propuseram um protocolo de comunicação V2V denominado Efficient Clustering V2V Routing Based on PSO (Particle Swarm Optimization) in VANETs (CRBP). Para tanto, o CRBP coleta informações tais como posição, velocidade e direção dos veículos para formar os agrupamentos e escolher um líder de cada agrupamento formado. Em seguida, é utilizado o filtro de partículas para selecionar os veículos retransmissores entre a origem e destino da mensagem. Entretanto, o veículo líder do agrupamento é responsável pela fusão dos dados coletados e pela computação da rota dentro de seu agrupamento. Com isso, o sistema depende que o veículo líder passe o maior tempo possível ativo. Isso compromete o funcionamento do protocolo uma vez que terá que haver uma nova escolha de líder e o algoritmo do filtro de partícula tem que ser novamente computado para as novas características da rede. Diferente do CRBP, o LEARN não depende da permanência de veículos específicos para manter a eficiência do protocolo.

Akabane et al. [Akabane et al. 2016] desenvolveram o protocolo ContextAware Routing (CARRO) que explora o conhecimento do contexto geográfico para a disseminação de dados nas VANETs em cenários urbanos e rodoviários. O CARRO é baseado no DRIVE [Villas et al. 2014], o qual seleciona os veículos localizados em uma região geográfica de alta prioridade para encaminhar uma mensagem. Para criar um conhecimento de topologia de rede sobre os veículos vizinhos, cada veículo transmite beacons periodicamente com sua posição, velocidade e direção. Quando CARRO detecta o 
problema de partição de rede, o mecanismo de armazenamento e transporte é usado. No entanto, esse mecanismo pode aumentar o atraso da rede. Além disso, o grande número de beacons necessários para o funcionamento do CARRO aumenta a sobrecarga da rede.

Costa et al. [da Costa et al. 2019] propuseram um protocolo de disseminação de dados baseado em métricas de rede complexas, denominado DDRX. No DDRX, os veículos mantêm um conhecimento local de seus vizinhos de 1 e 2 saltos, que são usados para construir um subgrafo. Usando a centralidade de intermediação e centralidade de grau, o DDRX seleciona os melhores veículos para retransmitir a mensagem. O DDRX fornece disseminação de dados com baixa sobrecarga e atraso na rede, maximizando a cobertura e minimizando o número de colisões de pacotes. Como foi utilizado métricas de rede complexas é necessário mais processamento de dados e uma maior quantidade de mensagens de controle que o LEARN. Neste caso é necessário conhecimento dos nós com 2 saltos de distância. Está solução também fica comprometida em redes mais espaças e não leva em consideração a localização do no destino assim tendo que disseminar a mensagem em toda rede diferentemente do LEARN.

Outro trabalho que assemelha com esta proposta e que foi utilizado para comparar com esta pesquisa foi apresentada por Lourenço et al. [Lourenço et al. 2019]. Em [Lourenço et al. 2019], é apresentado o DESTINy (DEcentralized System for Traffic Management), uma solução distribuída para gerenciamento de tráfego que usa o poder computacional do veículo para detectar congestionamentos e calcular novas rotas quando necessário. Para tanto, cada veículo, por meio de uma infraestrutura auxiliar de comunicação, recebe informações sobre as características de tráfego das vias próximas. Tal infraestrutura é responsável por atualizar e enviar as informações da estrada dentro da região definida. Periodicamente, os veículos solicitam as características da via para a infraestrutura mais próxima e, com base nessas informações recebidas, o veículo realiza o processo de roteamento. Embora o processamento das estradas seja distribuído entre os veículos, a infraestrutura ainda tem a responsabilidade de coletar e agregar as características das estradas para enviar essas informações aos veículos. O processo usado na comunicação V2V do DESTINy é baseado na distância para o destino. Entretanto a metodologia de distância para o destino pode falhar pois o veículo mais próximo pode levar a uma partição da rede e diferentemente do LEARN, a solução não possui nenhum método de recuperação caso isso ocorra.

Além das características supracitadas, diferentemente dos trabalhos relacionados, o LEARN implementa um mecanismo de recuperação de transmissão. O mecanismo se baseia na técnica backtracking, onde na presença de falha o protocolo volta um passo para tentar novamente outro caminho para aumentar a taxa de entrega e a cobertura sem sobrecarregar a rede com mensagens desnecessárias.

\section{LEARN: protocoLo de rotEamento para vAnet baseado em setoRização hexagoNal}

Esta seção apresenta o LEARN, um protocoLo de roteamento para VANET baseado em setorização hexagonal com múltiplo saltos. O LEARN seleciona os veículos de retransmissão com base nas mensagens disseminadas nas VANETs. Para tanto, é considerada a posição geográfica do veículo de destino e a posição dos veículos vizinhos em relação ao veículo retransmissor. Com isso, os veículos mantêm o conhecimento local de seus 


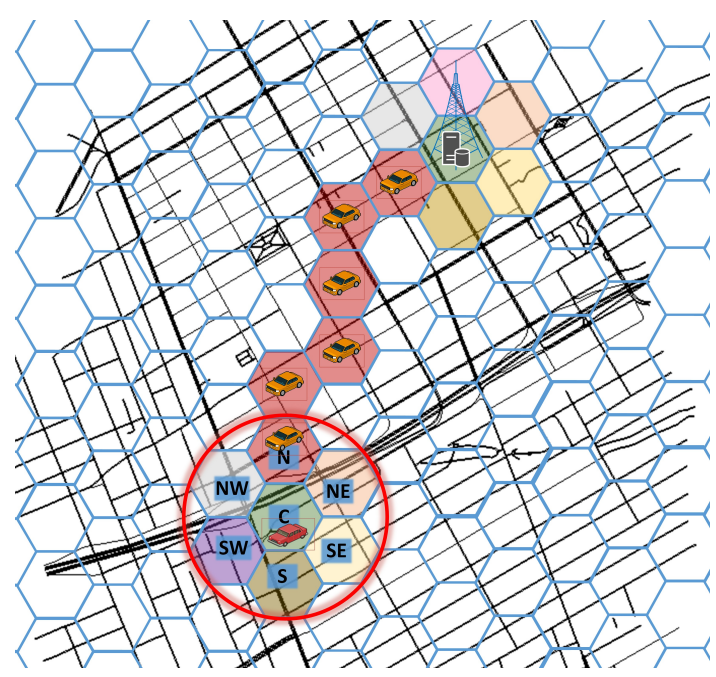

Figura 1. Setorização hexagonal e ilustração do funcionamento do LEARN.

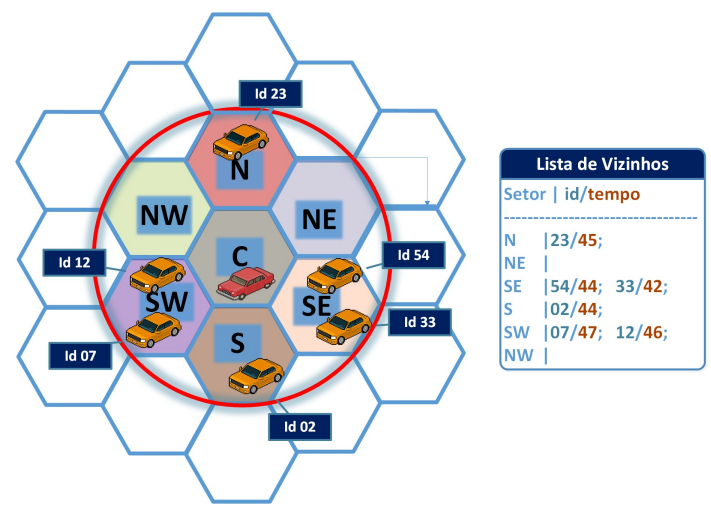

Figura 2. Setores com veículos vizinhos e lista de vizinhos.

vizinhos obtido por meio de beacons enviados pelos veículos. No LEARN, o cenário é discretizado em setores com formato hexagonal, Figura 1. Ao usar essa estratégia, o veículo que enviou a mensagem é associado ao setor da sua posição atual na base local de conhecimento do veículo receptor, Figura 2. No processo de encaminhamento do LEARN, as mensagens são transmitidas a um veículo do setor adjacente mais próximo ao nó de destino. Este processo é repetido até alcançar o destino (hexágonos vermelhos na Figura 1). Observe que a métrica para escolha do próximo setor é o menor caminho, entretanto outra métrica como ocupação dos setores pode ser facilmente implementada.

Um serviço ITS infraestruturado de sugestão de rota necessita de uma distribuição de RSU no cenário para obter uma cobertura de comunicação nas áreas em que o serviço atuará. Os serviços de sugestão de rota geralmente funcionam da seguinte maneira. O veículo solicita uma rota alternativa para o servidor por meio da RSU localizada em sua cobertura de comunicação. Em seguida, o serviço calcula a nova rota e responde ao veículo. Diferente dessa estratégia, vale salientar que o LEARN diminui os custos de implantação dos serviços uma vez que a comunicação é feita em múltiplos saltos, exigindo menos infraestrutura para o funcionamento do serviço. Com a aplicação do LEARN, é possível adicionar RSU apenas em pontos estratégicos do cenário (por exemplo, vias de alta densidade ou cruzamento) para servir o maior número de veículos com o menor número de saltos entre os veículos e as RSUs.

O LEARN setoriza o mapa em hexágonos como metodologia para escolher o nó retransmissor. O hexágono é um polígono regular que possui propriedades desejáveis para o LEARN. No LEARN, os hexágonos podem cobrir uniformemente o plano sem sobreposição, e é possível circunscrever o hexágono e seus vizinhos adjacentes regularmente [Li et al. 2017]. O centro do hexágono é compartilhado com o centro do círculo. Como definição para o LEARN, o raio para circunscrever o hexágono e seus seis vizinhos adjacentes é calculado de forma que o veículo esteja localizado no setor central (Figura 1 setor C) possa se comunicar com qualquer veículo localizado nos setores vizinhos (Figura 1 setores $\mathrm{N}, \mathrm{NE}, \mathrm{SE}, \mathrm{S}, \mathrm{SW}, \mathrm{NW}$ ). Para isso, baseado nas propriedades do hexágono, formulamos a Equação (1) que relaciona o raio de comunicação do veículo com o raio do 
círculo que circunscreve um hexágono e os hexágonos adjacentes (Figura 2 círculo vermelho). A largura do hexágono é (w) e a cobertura é o raio de comunicação do veículo.

$$
w \approx 0.5547 * \text { cobertura }
$$

A seguir será apresentada a modelagem do LEARN.

\subsection{Descoberta e conhecimento da vizinhança}

O LEARN usa uma On-Board Unit (OBU) para adquirir conhecimento dos veículos vizinhos que estão em um salto por meio dos beacons, os quais contêm informações do veículo tais como ID e sua localização atual. A OBU é um dispositivo equipado nos veículos com poder de processamento e permite comunicações DSRC/802.11p [Brennand et al. 2019] com outras OBUs e RSUs. Ao receber um beacon, o protocolo armazena o ID do veículo, a sua localização e a data/hora de recebimento. Os dados são armazenados em uma das 6 listas (N, NE, SE, S, SW, NW) correspondente ao setor que o veículo transmissor está localizado em relação ao receptor, como apresentado na Figura 2.

As listas com as informações são usadas para escolher o próximo salto na transmissão da mensagem. A lista é periodicamente atualizada devido a mobilidade do veículo. O período foi estimado de acordo com o tempo médio necessário para o veículo mudar de setor baseado na velocidade média do cenário. Por exemplo, em um cenário com velocidade média de $54 \mathrm{~km} / \mathrm{h}$, o tempo para o veículo sair do setor com tamanho de 160 metros em que está localizado no centro será de aproximadamente 5 segundos. Vários trabalhos estimam uma janela de atualização semelhante que geraram resultados satisfatórios [Tonguz et al. 2010, Villas et al. 2014, da Costa et al. 2019].

\subsection{Processo de disseminação}

No LEARN, o processo de disseminação das mensagens é realizado escolhendo os veículos intermediários mais adequados para encaminhar a mensagem para o veículo de destino. O LEARN tem dois componentes de comunicação sem fio em seu ecossistema, RSUs e veículos. As RSU recebem mensagens direcionadas a ela originadas de um veículo. Considerando isso, a RSU encaminha a mensagem recebida para o servidor do serviço. Em seguida o serviço envia uma mensagem de resposta para o veículo de origem. Mensagens comuns a serem enviadas pelos serviços de sugestão de rota são as solicitações de uma rota alternativa e mensagens periódicas contendo informações sobre as condições das vias.

O LEARN possui duas metodologias de roteamento das mensagens relacionadas a origem e destino da mensagem enviada. Quando a mensagem tem como destino um veículo em que se conhece apenas sua localização geográfica o LEARN aplica uma metodologia de escolhas dos veículos para retransmissão. No caso do serviço de sugestão de rota este caso acontece quando o veículo solicita a RSU uma rota ou envia as condições da via. O segundo caso é quando uma RSU envia uma mensagem de resposta (por exemplo, nova rota). O LEARN armazena todos os veículos retransmissores durante o envio das mensagens, utilizando a lista no processo de transmissão. 


\subsection{Roteamento de mensagens endereçadas a RSU}

No LEARN, quando um veículo prepara uma mensagem endereçada à RSU, é verificado se a RSU está em sua cobertura de comunicação. Nesse caso, a mensagem é enviada diretamente. Caso contrário, o LEARN usa a comunicação de múltiplo saltos. Para tanto, o LEARN usa o conhecimento prévio sobre a localização geográfica das RSUs e o conhecimento dos nós vizinhos. A Figura 1 apresenta o cenário discretizado, bem como o comportamento do LEARN. Como pode ser visto, o veículo do setor $C$ deseja enviar uma mensagem para a RSU mais próxima. Ao iniciar o processo de roteamento, é necessário verificar quais setores vizinhos possuem pelo menos um veículo e quais setores foram visitados para evitar o looping. Portanto, para encontrar o melhor caminho para o destino, o protocolo faz um roteamento de alto nível usando o algoritmo do caminho mais curto (neste caso, Dijkstra) no grafo que representa os setores discretizados no cenário. Quando o próximo setor é escolhido, um veículo localizado neste setor é selecionado e a mensagem é endereçada a ele. Este mecanismo é executado a cada salto pelo respectivo veículo retransmissor até alcançar o destino. O Algoritmo 1 formaliza este protocolo.

O Algoritmo 1 tem como ponto inicial o setor em que o veículo está localizado e como ponto final o setor da RSU de destino. Os outros parâmetros de entrada são a lista de vizinhos, a lista de hexágonos e suas posições, a lista de ID e posições das RSUs, ID do veículo, posição e setor atual (linhas 1 a 5, Algoritmo 1). Para evitar que o LEARN escolha um setor que não possua veículos ou crie um loop quando a mensagem é roteada para o destino, um setor pode ser removido do grafo de setores em alguns casos. $\mathrm{O}$ primeiro caso é apresentado na linha 7 do Algoritmo 1, em que é verificado quais setores vizinhos (lista_vizinho) contêm pelo menos um veículo ativo. Setores vizinhos que não possuem veículos, são removidos temporariamente do grafo do setor. O segundo caso (linha 12, Algoritmo 1), todos os setores contidos na lista visitados_setores recebidos na mensagem a ser retransmitida são temporariamente removidos do grafo (Gtmp). Com isso, o próximo salto é escolhido com base no algoritmo de caminho mais curto entre o setor atual e o setor de destino usando o algorítimo de menor caminho no grafo de setores, linha 20 do Algoritmo 1.

Com o setor escolhido, é verificado se mais de um veículo é candidato a retransmissor neste setor. Em caso afirmativo, o veículo com o beacon mais recente é escolhido (linha 16, Algoritmo 1). Finalmente, os campos da mensagem são atualizados (linhas 17 - 20, Algoritmo 1). Durante o processo de retransmissão da mensagem, observe que os IDs dos veículos retransmissores e dos setores visitados são armazenados nas listas veiculos_visitado e setores_visitados, respectivamente (linhas 19, 20, Algoritmo 1). Além de serem utilizadas neste método, essas listas são utilizadas na metodologia que o LEARN usa no processo de resposta da mensagem, como será apresentada na Subseção 3.4. Este processo se repete até mensagem chegar ao destino.

Em caso de erros de retransmissão, o LEARN possui um modo de recuperação. A técnica de recuperação é semelhante à técnica de backtracking[Ginsberg 1993] em que se um nó retransmissor não estiver nos setores vizinhos com veículos elegíveis para retransmissão, o veículo anterior tentará encaminhar a mensagem através do segundo setor mais próximo, caso exista. Para isso, um cronômetro é acionado a cada mensagem enviada e o veículo ficará escultando o canal de transmissão. Se o veículo não escutar a retransmissão neste período, a mensagem é atualizada (o recovery_tag é adicionada 1, o setor anterior- 


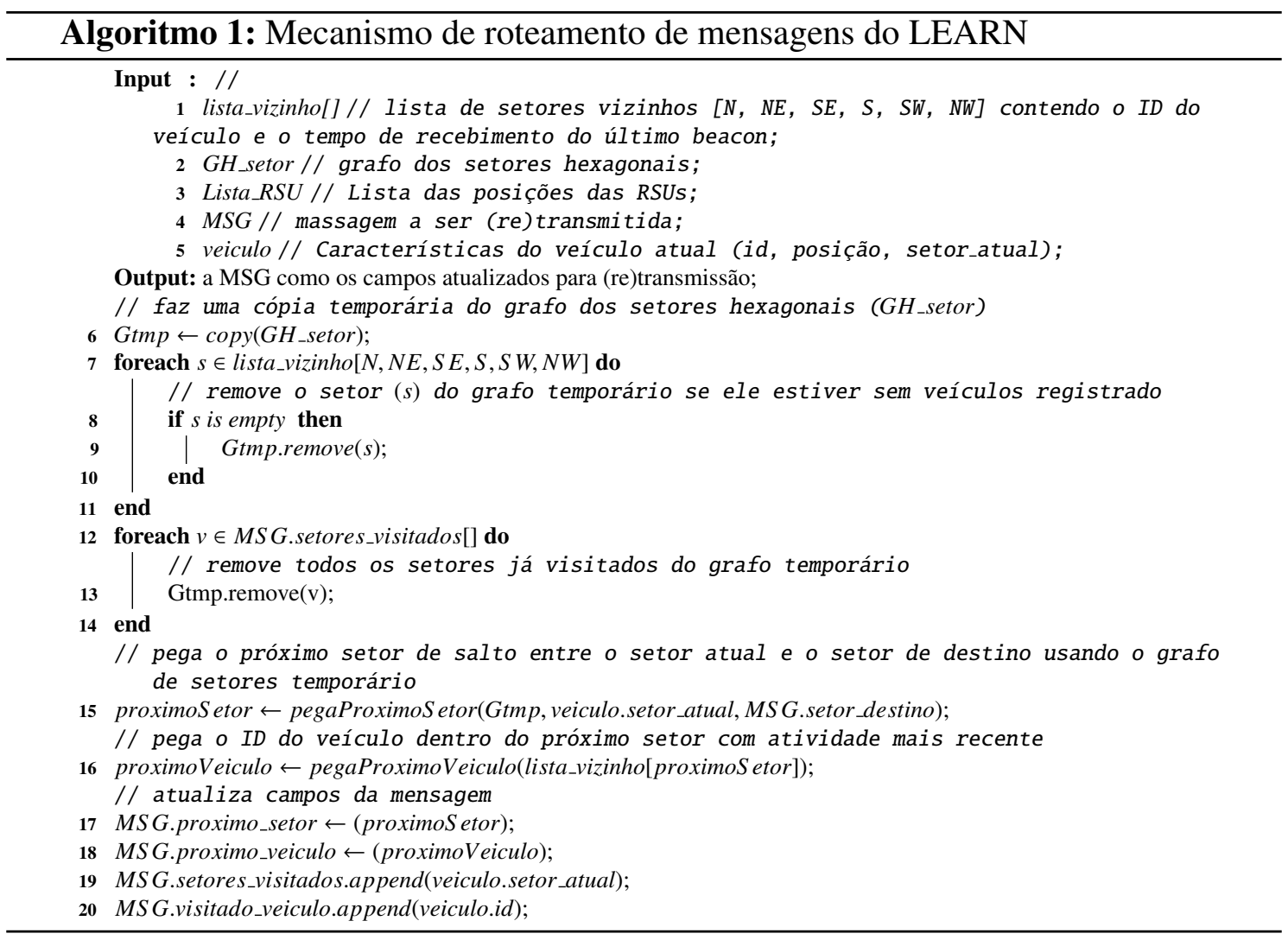

mente utilizado para transmissão é colocado na lista setores_visitados) e o processo de escolha um novo setor de próximo salto para retransmissão é iniciado (Algoritmo 1). O campo recovery_tag é usado para limitar o número de passos do backtracking.

\subsection{Encaminhamento de mensagens de resposta}

Este mecanismo de roteamento é usado quando os veículos retransmissores necessários para alcançar o nó de destino são conhecidos. O mecanismo é usado quando uma mensagem de resposta para o veículo é enviada pela RSU. Como o tempo de resposta do serviço é rápido o suficiente em comparação com as mudanças na topologia da rede, a mensagem de resposta do veículo solicitante usa a lista de veículos retransmissores (MSG.Veiculo_visitados) contida na mensagem de solicitação.

Assim, a transmissão e feita diretamente entre os nós contida na lista até alcançar o veículo de destino. A cada retransmissão da mensagem de resposta, o LEARN remove o último ID da lista e o último setor visitado. Além disso, a cada transmissão o LEARN ativa o cronômetro definido para $100 \mathrm{~ms}$, que é o tempo para alterações de dois canais no protocolo 802.11 p para escultar se o próximo veículo, caso não seja o destino final, retransmitiu a mensagem. Esta metodologia é aplicada devido à probabilidade de uma falha de transmissão (por exemplo, mudança de topologia). Caso ocorra, o LEARN entra no modo de recuperação ativando o Algoritmo 1, passando como entrada o veículo e setor atual/destino e a lista dos veículos e setores visitados. A seguir é apresentada a validação do LEARN. 


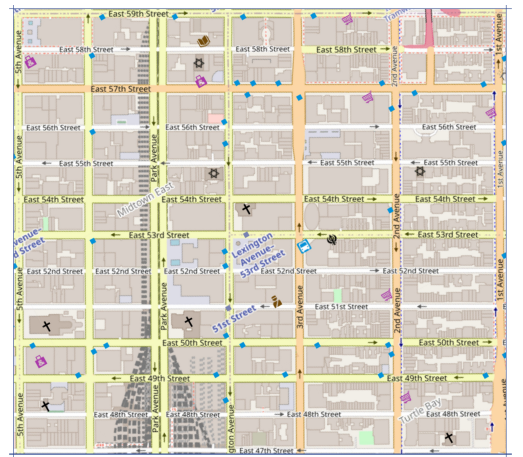

Figura 3. Topologia do cenário de Nova York $\left(1 \mathrm{~km}^{2}\right)$.

\begin{tabular}{|l||l|}
\hline Parâmetros & Valores \\
\hline \hline Potência de transmissão & $2.2 \mathrm{~mW}$ \\
Cobertura & $300 \mathrm{~m}$ \\
Taxa de transferência & $18 \mathrm{Mbit} / \mathrm{s}$ \\
Intervalo das mensagens & $4 \mathrm{~s}$ \\
Checagem de falha de transmissão & $100 \mathrm{~ms}$ \\
Rotas alternativas (k) & 3 \\
Veículos $/ \mathrm{km}^{2}$ & $50,150,500$ \\
Intervalo de confiança & $95 \%$ \\
\hline
\end{tabular}

Tabela 1. Parâmetros utilizados na simulação.

\section{Avaliação de Desempenho}

Nesta seção, é apresentada a avaliação de desempenho do LEARN, comparando-o com DESTINy e o Flooding. Para tanto, a validação foi dividida em duas etapas: (i) Avaliação da comunicação versus densidade para avaliar o impacto de diferentes densidades de veículos; e (ii) Avaliação da área de cobertura para analisar o impacto da cobertura do cenário variando o número de RSU e a relação com a densidade do cenário. A seguir, será apresentado o cenário de simulação modelado, bem como as métricas utilizadas e os parâmetros selecionados.

\subsection{Cenário de simulação}

O simulador OMNeT $++5^{1}$, foi utilizado para avaliar o LEARN. Para simular o tráfego e mobilidade dos veículos, foi utilizado o simulador SUMO 0.25.02. Ainda, foi utilizado o Framework Veins $4.3^{3}$ para a rede veicular e o IEEE 802.11p e o IEEE 1609.4 DSRC/WAVE como modelo de atenuação de sinal que considera obstáculos. Os experimentos consideraram uma área de $1 \mathrm{~km}^{2}$ da cidade de Nova York, EUA, como apresentado na Figura 3. A configuração do cenário possui uma densidade de veículos variando em 50,150 e 500 veículos $/ \mathrm{km}^{2}$. A cobertura do cenário pelas RSUs está descrita nos respectivos experimentos. Os experimentos simulam o comportamento de um serviço de sugestão de rota em que uma rota é solicitada e a RSU (ou servidor) responde com a rota sugerida. Para avaliação justa, uma vez que todos os veículos estão no cenário, periodicamente todos os veículos enviam uma mensagem para a RSU mais próxima. Quando a RSU recebe uma mensagem, a RSU envia uma resposta ao veículo. Para evitar que todos os veículos enviem mensagens ao mesmo tempo, o cronômetro é adicionado com uma variável aleatória entre $0-1$ segundos.

Os parâmetros de rede para todas as simulações foram configurados para 18Mbit/s na camada MAC e a potência de transmissão para $2.2 \mathrm{~mW}$, resultando em uma cobertura de aproximadamente $300 \mathrm{~m}$ sob um modelo de propagação terrestre de dois raios [Sommer et al. 2011]. O intervalo de envio das mensagens definido em $4 \mathrm{~s}$ para todas as soluções. O intervalo de confiança foi de $95 \%$.

\footnotetext{
${ }^{1}$ http: //www . omnetpp.org

${ }^{2}$ http://sumo.dlr.de

${ }^{3}$ http://veins. car 2 x.org/
} 
As seguintes métricas foram utilizadas para avaliar o desempenho do LEARN: i) Pacotes retransmitidos que apresenta o número total de retransmissões necessárias para chegar ao destino; ii) Colisões que apresenta o número total de colisões por veículo; iii) Cobertura que apresenta a porcentagem de veículos que receberam resposta pelo menos uma vez durante o tempo de simulação; e iv) Mensagens recebidas que apresenta a porcentagem de mensagens enviadas que foram respondidas.

\subsection{Avaliação da comunicação versus densidade}

Para este experimento foi usada 1 RSU para obter cobertura parcial do cenário, avaliando, portanto, a eficiência das soluções. A Figura 4(a) apresenta o número de mensagens retransmitidas em função da densidade de veículos. O Flooding obteve o maior número de mensagens retransmitidas com mais de 135.000 mensagens no cenário com 500 veículos $/ \mathrm{Km}^{2}$. Isso ocorre pois o Flooding não possui um mecanismo de supressão de mensagens. O mecanismo o DESTINy, que se baseia na distância, reduz o número de mensagens em $70 \%$ em relação ao Flooding. Entretanto, muitas mensagens ainda são geradas até que a mensagem chegue ao destino, necessitando até $90 \%$ mais mensagens do que o LEARN com a mesma densidade de veículo. O LEARN possui um número médio de retransmissões por mensagem enviada próximo ao número de setores percorridos até o destino, mantendo assim o número de mensagens no sistema reduzido.

A Figura 4(b) apresenta a colisão de pacotes variando a quantidade de veículos. O LEARN manteve estável a quantidade de colisões em relação ao número de veículos com 120, 4 mensagens colididas para a densidade de 500 veículos $/ \mathrm{km}^{2}$. Observe que o número de colisões do Flooding (26.000 para a densidade de 500 veículos $/ \mathrm{km}^{2}$ ) cresce rapidamente quando comparado com o DESTINy (8.000 para a densidade de 500 veículos $/ \mathrm{Km}^{2}$ ) e com o LEARN. Isso está relacionado ao grande número de mensagens retransmitidas, como apresentada na Figura 4(a).

A Figura 4(c) apresenta a porcentagem de veículos que enviaram e receberam a resposta de pelo menos uma mensagem durante a simulação. O LEARN atingiu uma cobertura média de $98 \%$ devido a baixa colisão e o método de recuperação aplicado. O Flooding e DESTINy obtiveram uma redução significativa na cobertura com um aumento na densidade de veículos cobrindo apenas 33\% e 64\% dos veículos no cenário com uma densidade de 500 veículos $/ \mathrm{Km}^{2}$. Esses valores são corroborados pelo alto número de colisões dessas soluções. Portanto, o LEARN pode atingir todo o ambiente monitorado.

A Figura 4(d) apresenta os percentuais de mensagens enviadas e respondidas corretamente durante a simulação. No cenário de baixa densidade (50 veículos $/ \mathrm{km}^{2}$ ), a porcentagem de mensagens respondidas foi de $79 \%$ para o LEARN, 65\% para o Flooding e $60 \%$ para o DESTINy. Com o aumento da densidade, o LEARN obteve o percentual médio de entrega em 74\% enquanto o Flooding e o DESTINy obtiveram uma grande redução, chegando a estar abaixo de $20 \%$ para a densidade de 500 veículos $/ \mathrm{km}^{2}$. Isso ocorreu devido ao grande número de colisões de mensagens geradas. Assim, o LEARN mostrou-se eficiente tanto em um cenário esparso quanto em um cenário mais denso.

\subsection{Avaliação da Área de Cobertura}

Para avaliar o impacto da cobertura do cenário, alterou-se o número de RSU (1, 4 e 8 ) e a densidade do cenário $\left(50,150,500\right.$ veículos $\left./ \mathrm{km}^{2}\right)$. Com 8 RSUs, o LEARN fornece 


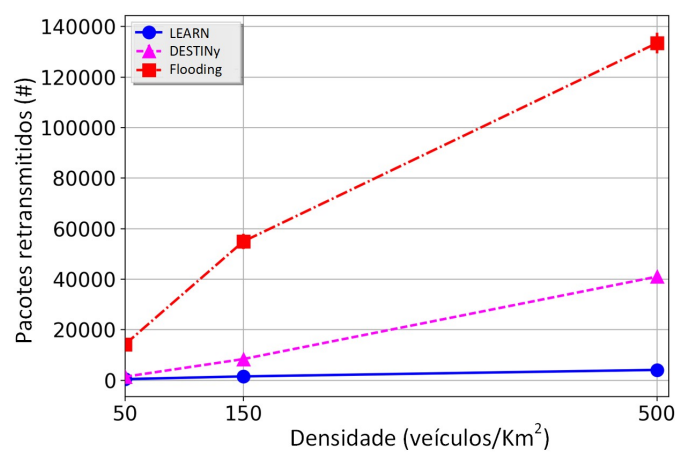

(a) Total de pacotes retransmitidos.

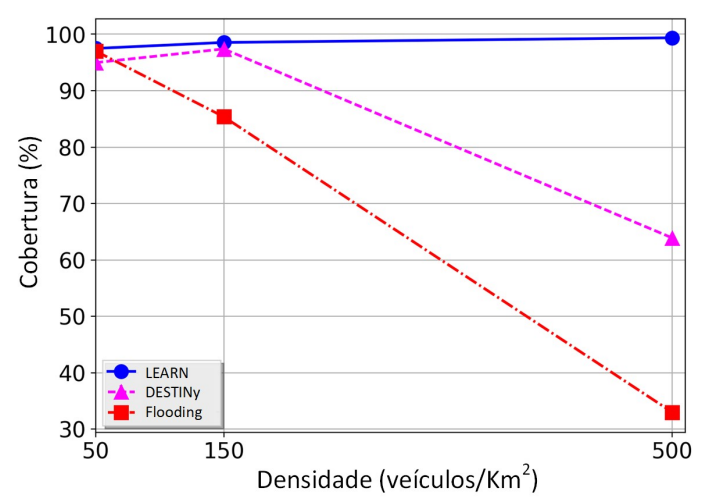

(c) Cobertura $(\%)$.

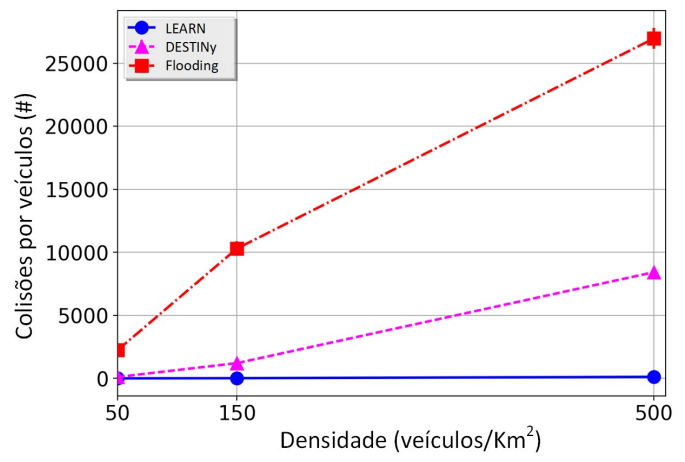

(b) Número de colisões por veículos.

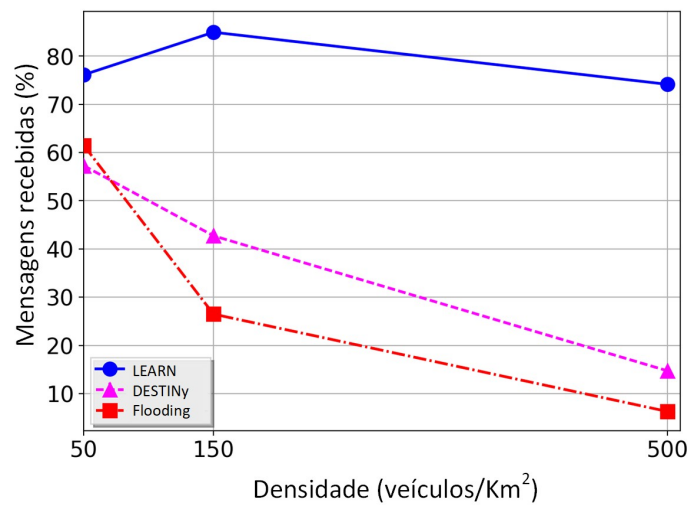

(d) Porcentagem de mensagens retornadas.

Figura 4. Desempenho dos protocolos LEARN, DESTINy e Flooding variando a densidade de veículos.

cobertura total do cenário. A Figura 5(a) apresenta o número de pacotes retransmitidos. Percebe-se, que como esperado, o número de mensagens retransmitidas aumenta com a densidade de veículos e a redução do número de RSU. A metodologia de setorização aplicada e roteamento interativo do LEARN mantendo baixo o número de mensagens no sistema, mesmo com uma alta densidade de veículos e com cobertura de RSU reduzida.

A Figura 5(b), nota-se que o número de colisões de pacotes aumenta com a necessidade de usar comunicação de múltiplo saltos, pois mais mensagens são necessárias para alcançar o destino que estará mais distante. ainda, observe que quanto maior a cobertura do RSU, menos mensagens são necessárias para alcançar o destino.

O LEARN possui eficiência na cobertura, conforme apresentado na Figura 5(c). Quando a densidade do veículo é baixa, problemas como desconexão da rede reduzem a área de cobertura. Esse problema pode ser reduzido aplicando metodologia de transmissão story-carry-forward. Entretanto, como o LEARN foi desenvolvido visando os serviços de controle de congestionamento, o LEARN não implementa esta metodologia uma vez que regiões com baixa densidade de veículos apresentam baixa probabilidade de apresentar congestionamento de tráfego.

A porcentagem de resposta para mensagens enviadas (vide Figura 5(d)), é maior quando o número de RSU é maior. Em relação ao uso de 1 RSU, quando a densidade de veículos é de 50 veículos $/ \mathrm{km}^{2}$, o número de mensagens recebidas é de $76 \%$. Este valor é 
influenciado pelo problema da partição da rede. Para a densidade é de 500 veículos $/ \mathrm{km}^{2}$ se obteve $74 \%$ de mensagens retornadas. Isto se deve à influência gerada pela colisão de pacotes conforme apresentado na Figura 5(b). Note o pico que ocorre na densidade de 150 veículos $/ \mathrm{km}^{2}$ no cenário com uma RSU. Isso se deve ao fato que para esta densidade o cenário possui um bom número de veículos para evitar a partição da rede, mas não muitos para gerar muitas colisões de pacotes assim reduzindo a taxa de entrega.

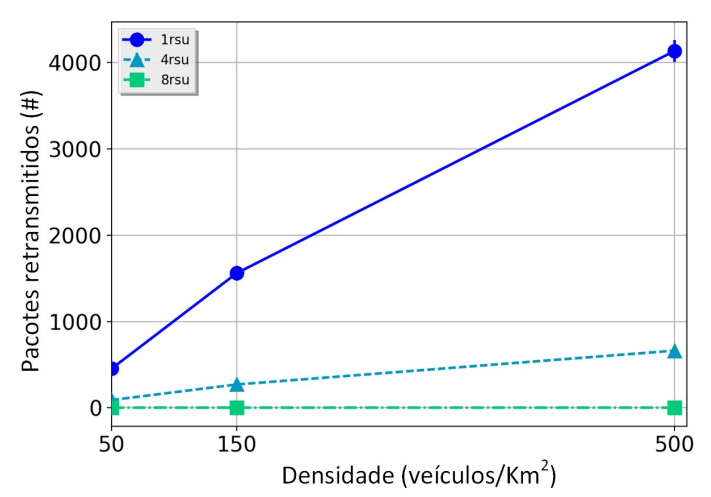

(a) Total de pacotes retransmitidos.

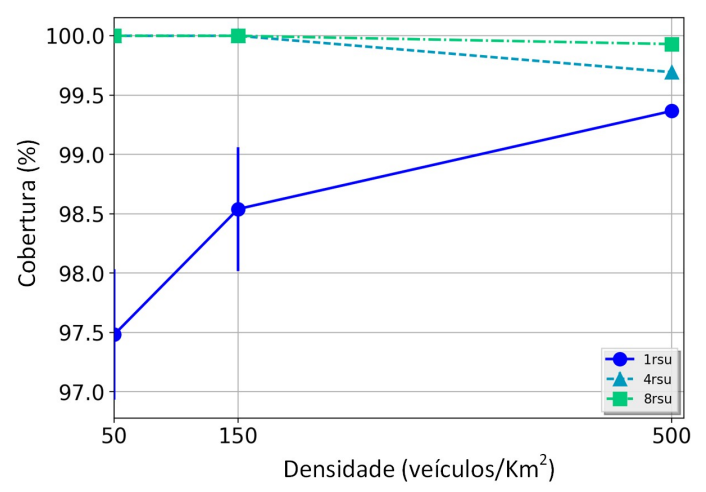

(c) Cobertura (\%).

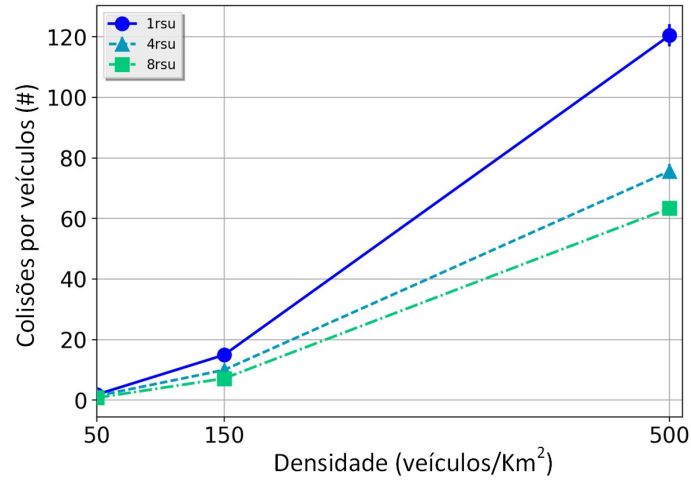

(b) Número de colisões por veículos.

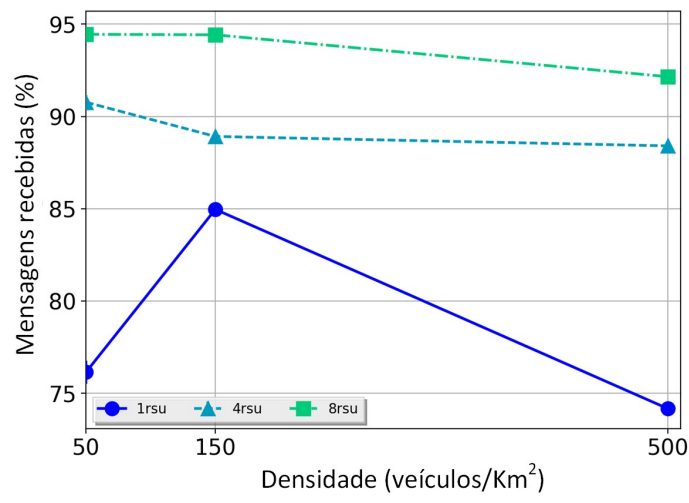

(d) Porcentagem de mensagens retornadas.

Figura 5. Desempenho dos protocolo LEARN variando a cobertura do cenário.

\section{Conclusão}

Este artigo apresentou o LEARN, um protocolo de comunicação múltiplo salto distribuído para VANET. O protocolo múltiplo salto proposto, de forma eficiente, permite que o veículo se comunique com uma RSU que não está em nossa cobertura permitindo assim se comunicar com o serviço na infraestrutura de forma eficiente. O protocolo setoriza o cenário em formato hexagonal. Dessa forma, a mensagem é encaminhada entre a origem e o destino utilizando os setores ocupados por veículos. O serviço foi desenvolvido pensando exclusivamente em serviços de sugestão de rota para veículos onde o veículo requisita uma nova roa a um serviço localizado em uma infraestrutura. Vários experimentos foram executados para mostrar a eficiência do LEARN. Esses experimentos mostraram que a colisão de pacotes do LEARN permaneceu baixa com o aumento na densidade do cenário tendo até $95 \%$ menos colisões do que as outras soluções. Comportamento semelhante também foi observado para métricas de cobertura de recebimento e 
pacotes transmitidos. Como trabalho futuro, planejamos realizar experimentos considerando diferentes cenários. Realizar mais experimentos com serviços de roteamentos da literatura. Otimizar o protocolo para usar nós móveis de computação névoa (por exemplo, ônibus), removendo ainda mais a necessidade de cobertura do cenário por infraestrutura. Propor uma extensão do protocolo para abranger todos os tipos de serviços adicionando metodologias como story-carry-forward e tunelamento de mensagens entre regiões.

\section{Referências}

Akabane, A. T., Pazzi, R. W., Madeira, E. R., and Villas, L. A. (2016). Carro: A contextawareness protocol for data dissemination in urban and highway scenarios. In 2016 8th IEEE Latin-American Conference on Communications (LATINCOM), pages 1-6. IEEE.

Bao, X., Li, H., Zhao, G., Chang, L., Zhou, J., and Li, Y. (2020). Efficient clustering v2v routing based on pso in vanets. Measurement, 152:107306.

Brennand, C. A. R. L., Boukerche, A., Meneguette, R., and Villas, L. A. (2017). A novel urban traffic management mechanism based on fog. In 2017 IEEE Symposium on Computers and Communications (ISCC), pages 377-382.

Brennand, C. A. R. L., Filho, G. P. R., Maia, G., Cunha, F., Guidoni, D. L., and Villas, L. A. (2019). Towards a fog-enabled intelligent transportation system to reduce traffic jam. Sensors, 19(18).

Chen, S., Kuhn, M., Prettner, K., and Bloom, D. E. (2019). The global macroeconomic burden of road injuries: estimates and projections for 166 countries. The Lancet Planetary Health, 3(9):e390 - e398.

Correa, C., Ueyama, J., Meneguette, R. I., and Villas, L. A. (2014). Vanets: An exploratory evaluation in vehicular ad hoc network for urban environment. In 2014 IEEE 13th International Symposium on Network Computing and Applications, pages 45-49.

da Costa, J. B., de Souza, A. M., Rosário, D., Cerqueira, E., and Villas, L. A. (2019). Efficient data dissemination protocol based on complex networks' metrics for urban vehicular networks. Journal of Internet Services and Applications, 10(1):15.

da Cunha, F. D., Villas, L., Boukerche, A., Maia, G., Viana, A. C., Mini, R. A. F., and Loureiro, A. A. F. (2016). Data communication in vanets: Protocols, applications and challenges. Ad Hoc Networks, 44:90-103.

De Souza, A. M., Brennand, C. A., Yokoyama, R. S., Donato, E. A., Madeira, E. R., and Villas, L. A. (2017). Traffic management systems: A classification, review, challenges, and future perspectives. International Journal of Distributed Sensor Networks, 13(4):1550147716683612.

Ginsberg, M. L. (1993). Dynamic backtracking. Journal of artificial intelligence research, $1: 25-46$.

H. Allen, K. M. and Stonehill, M. (2013). A summary of the proceedings from the united nations climate change conference in doha, qatar, and their significance for the land transport sector, copenhagen: Bridging the gap (btg) initiative.

Li, T., Xia, M., Chen, J., Gao, S., and De Silva, C. (2017). A hexagonal grid-based sampling planner for aquatic environmental monitoring using unmanned surface vehicles. 
In 2017 IEEE International Conference on Systems, Man, and Cybernetics (SMC), pages 3683-3688. IEEE.

Lourenço, M., Souza, F. S., Silva, C. M., Meneguette, R. I., and Guidoni, D. L. (2019). A hybrid $\mathrm{v} 2 \mathrm{i}$ and $\mathrm{v} 2 \mathrm{v}$ approach for urban traffic management in vehicular networks. In 2019 IEEE Latin-American Conference on Communications (LATINCOM), pages 1-6. IEEE.

Meneguette, R. I., De Grande, R., and Loureiro, A. (2018). Intelligent transport system in smart cities. Springer.

Organization, W. H. et al. (2018). Global status report on road safety 2018: Summary. Technical report, World Health Organization.

Rodrigues, D., Severino, J. N., Cristiani, A. L., Costa, F. T. M., Nakamura, L. H. V., and Meneguette, R. I. (2019). A low-cost vehicular infrastructure of monitoring and communicating information for intelligent transport systems.

Ros, F., Ruiz, P., and Stojmenovic, I. (2012). Acknowledgment-based broadcast protocol for reliable and efficient data dissemination in vehicular ad hoc networks. Mobile Computing, IEEE Transactions on, 11(1):33-46.

Schwartz, R. S., Barbosa, R. R., Meratnia, N., Heijenk, G., and Scholten, H. (2011). A directional data dissemination protocol for vehicular environments. Computer Communications, 34(17):2057-2071.

Silva, T. H., Viana, A. C., Benevenuto, F., Villas, L., Salles, J., Loureiro, A., and Quercia, D. (2019). Urban computing leveraging location-based social network data: A survey. ACM Comput. Surv., 52(1).

Sommer, C., German, R., and Dressler, F. (2011). Bidirectionally Coupled Network and Road Traffic Simulation for Improved IVC Analysis. IEEE Transactions on Mobile Computing, 10(1):3-15.

Srivastava, A., Prakash, A., and Tripathi, R. (2020a). An adaptive intersection selection mechanism using ant colony optimization for efficient data dissemination in urban vanet. Peer-to-Peer Networking and Applications, 13(5):1375-1393.

Srivastava, A., Prakash, A., and Tripathi, R. (2020b). Location based routing protocols in vanet: Issues and existing solutions. Vehicular Communications, 23:100231.

Texas Transportation Institute, D.L. Schrank, W. T. J. (2015). 2015 urban mobility scorecard, mobility report. last visited in December, 2018.

Tonguz, O., Wisitpongphan, N., and Bai, F. (2010). Dv-cast: A distributed vehicular broadcast protocol for vehicular ad hoc networks. Wireless Communications, IEEE, 17(2):47-57.

Villas, L. A., Boukerche, A., Maia, G., Pazzi, R. W., and Loureiro, A. A. (2014). Drive: An efficient and robust data dissemination protocol for highway and urban vehicular ad hoc networks. Computer Networks, 75, Part A(0):381 - 394.

Viriyasitavat, W., Tonguz, O., and Bai, F. (2011). UV-CAST: an urban vehicular broadcast protocol. IEEE Communications Magazine, 49(11):116-124. 